Please do not remove this page

RMIT

UNIVERSITY

\title{
Design of lattice structures with controlled anisotropy
}

Xu, Shanqing; Shen, Jianhu; Zhou, Shiwei; Huang, Xiaodong; Xie, Yimin

https://researchrepository.rmit.edu.au/esploro/outputs/9921862684801341/filesAndLinks?institution=61RMIT_INST\&index=null

Xu, S., Shen, J., Zhou, S., Huang, X., \& Xie, Y. (2016). Design of lattice structures with controlled anisotropy. Materials and Design, 93, 443-447. https://doi.org/10.1016/j.matdes.2016.01.007

Document Version: Accepted Manuscript

Published Version: https://doi.org/10.1016/j.matdes.2016.01.007

Repository homepage: https://researchrepository.rmit.edu.au

(c) 2016 Elsevier Ltd. All rights reserved.

Downloaded On 2023/04/26 22:49:51 +1000

Please do not remove this page 
Thank you for downloading this document from the RMIT Research Repository.

The RMIT Research Repository is an open access database showcasing the research outputs of RMIT University researchers.

RMIT Research Repository: http://researchbank.rmit.edu.au/

\author{
Citation: \\ Xu, S, Shen, J, Zhou, S, Huang, X and Xie, Y 2016, 'Design of lattice structures with \\ controlled anisotropy', Materials and Design, vol. 93, pp. 443-447.
}

See this record in the RMIT Research Repository at:

https://researchbank.rmit.edu.au/view/rmit:35766

Version: Accepted Manuscript

\title{
Copyright Statement:
}

(c) 2016. This manuscript version is made available under the CC-BY-NC-ND 4.0 license http://creativecommons.org/licenses/by-nc-nd/4.0/

Link to Published Version:

https://dx.doi.org/10.1016/j.matdes.2016.01.007 


\title{
Design of Lattice Structures with Controlled Anisotropy
}

\author{
Shanqing Xu, Jianhu Shen, Shiwei Zhou, Xiaodong Huang, and Yi Min Xie* \\ Centre for Innovative Structures and Materials, School of Engineering, RMIT University, GPO Box 2476, \\ Melbourne 3001, Australia
}

*Corresponding author: Yi Min Xie, School of Civil, Environmental and Chemical and Engineering, RMIT University, GPO Box 2476, Melbourne 3001, Australia. Tel. +61 39925 3655. mike.xie@rmit.edu.au

\begin{abstract}
Recent advances in additive manufacturing make it possible to fabricate periodic lattice structures with complex configurations. However, a proper design strategy to achieve lattice structures with controlled anisotropy is still unavailable. There is an urgent need to fill this knowledge gap in order to develop mechanical metamaterials with prescribed properties. Here we propose two different methodologies to design lattice structures with controlled anisotropy. As examples, we created two new families of lattice structures with isotropic elasticity and cubic symmetric geometry. The findings of this work provide simple and effective strategies for exploring lightweight metamaterials with desired mechanical properties.
\end{abstract}

Keywords: Metamaterials, Lattice structures, Anisotropy, Homogenization

\section{Introduction}

Cellular structures are widely adopted as biological organisms in nature through generations of natural selection, e.g., wood and bones (Gibson and Ashby, 1991), sponge and diatom (Round et al., 1990; Bäeuerlein et al., 2007). Cellular materials including honeycomb and foam-like structures have high strength-to-weight ratios and other superior properties (Gibson and Ashby, 1991). Thus artificial cellular materials were developed and fabricated in the past decades on a large scale for a broad range of applications such as energy absorption (Evans et al., 2010), energy storage (Liu et al., 2010), structural components (Wadley, 2006), catalyst carrier and biomaterials (Hutmacher, 2000; Karageorgiou and Kaplan, 2005). A majority of manmade cellular materials are periodic structures due to their stable mechanical properties and ease for mass production. Various periodic structures have been designed in the past to accommodate the increasing demands of applications requiring specific mechanical properties. The increasing advancement of various additive manufacturing technologies makes it possible to fabricate complex structures which cannot be processed by conventional technologies (Gibson et al., 2010; Guo and Leu, 2013; Giannitelli, 2014; Murphy and Atala, 2014). The challenge is therefore finding the optimal design of structures. A typical case is the design of bone implants. The stiffness of the artificial bone is anisotropic and has to be delicately designed as porous structures to match the neighbouring natural bones and avoid the stress shielding, which may cause significant malfunction after long service. Analyses on natural bone show that the spatial distribution of Young's modulus is smooth without sharp increase or decrease in certain directions. However, most existing periodic bone implants focused on simply reducing the stiffness (Gurtner and Durand, 2014; Sallica-Leva et al., 2013; Murr et al., 2010; Ahmadi et al., 2014; Yang et al., 2013; Parthasarathy et al., 2010; Chantarapanich et al., 2012; Challis et al., 2010; Wieding et al., 2014; Heinl et al., 2008) with very few seeking for structures with isotropic or controlled anisotropic elasticity (Gurtner and Durand, 2014; Ahmadi et al., 2014; Yang et al., 2013; Challis et al., 2010). Besides, most designs of bone implants were not truss-like lattice and had complex internal structures since they were obtained through topological optimization techniques (Yang et al., 2013; Challis et al., 2010; Xie et al., 2014). 
Lattice structures are structurally simple and widely used not only as biomaterials, but also as other components in civil, aerospace and mechanical engineering. Studies on design and manufacturing lattice structures are becoming increasingly important due to the attractiveness of ultra-stiff and ultra-strong metamaterials with exceptional properties (Zheng et al., 2014; Meza et al., 2014; Schaedler et al., 2011). Design of these structures with directionally controlled mechanical properties is of critical importance in various applications. However, systematic and effective methodologies for designing such lattice structures with controlled anisotropy are still unavailable. A conventional method to create a nearly isotropic lattice structure is to select proper representative unit cells from 3D architecture libraries and make modifications on them to achieve goals (Ahmadi et al., 2014; Chantarapanich et al., 2012). This procedure usually involves large amount of work on finite element modelling to evaluate the stiffness of structures in various loading directions through trial-and-error methods, which is tedious and time-consuming. In this work, we propose methodologies from a new perspective which can effectively and efficiently build lattice structures with controlled anisotropy. To achieve this, we first solve the problem of characterization and evaluation of direction-dependent Young's modulus spatial distribution of lattice structures. Previously the Young's modulus spatial surface was used to study the anisotropy of monocrystallines (Nye, 1985; Meyers and Chawla, 2009). Similarly, it is possible to expand the method to represent the spatial distribution of Young's modulus for lattice structures, if the effective Young's modulus of these structures could be derived. Fortunately, the homogenization theory (Steven, 1997; Tan et al., 2000) makes this possible since it can accurately approximate the non-continuum periodic composites to continuum ones. Therefore, by combining the homogenization method and the 3D representation of Young's modulus, it becomes easy and straightforward to assess and analyse the representative unit cells. Built upon this, we find ways to design novel lattice structures with controlled anisotropy. These design methodologies will be useful for designing bone implants with desired mechanical properties and for creating the next generation ultra-stiff and ultra-strong metamaterials.

\section{Methods and calculation}

In this work, a simple and straightforward numerical homogenization technique proposed by Steven (Steven, 1997; Tan et al., 2000) was used to obtain the effective stiffness matrix of the non-continuum, periodic lattice structures. In Hooke's law, $\sigma_{i j}=C_{i j k l} \varepsilon_{k l}$, unknown constants of the forth-order stiffness matrix $\mathbf{C}$ can be reduced from 81 to 21 due to the symmetry in orthogonal system. In numerical realization (Steven, 1997; Tan et al., 2000; Xie et al., 2013), each time one strain component was set to unit whereas the rest five as zero, e.g., Eq. (1).

$$
\text { Input: }\left\{\begin{array}{l}
\varepsilon_{11} \\
\varepsilon_{22} \\
\varepsilon_{33} \\
\varepsilon_{23} \\
\varepsilon_{31} \\
\varepsilon_{12}
\end{array}\right\}=\left\{\begin{array}{l}
1 \\
0 \\
0 \\
0 \\
0 \\
0
\end{array}\right\} \text { output: }\left\{\begin{array}{l}
\sigma_{11} \\
\sigma_{22} \\
\sigma_{33} \\
\sigma_{31} \\
\sigma_{12}
\end{array}\right\}=\left\{\begin{array}{l}
C_{11} \\
C_{21} \\
C_{31} \\
C_{41} \\
C_{51} \\
C_{61}
\end{array}\right\}
$$

In this approach, the unit strain was expressed as a prescribed displacement on the boundary so that the corresponding stresses could be determined from reaction forces in finite element analyses. Therefore, six finite element analyses were used to determine all the components of the stiffness matrix. Two types of boundary conditions were involved in terms of the strain type, i.e., normal strain and shear strain (Tan et al., 2000). Take a normal strain $\varepsilon_{x}\left(\varepsilon_{11}\right)$ for instance, the boundary conditions were defined by 


$$
\begin{aligned}
& \left.\Delta l_{x}\right|_{x=l_{x}}=0.001 l_{x} \\
& \left.\Delta l_{x}\right|_{x=0}=\left.\Delta l_{y}\right|_{y=l_{y}}=\left.\Delta l_{y}\right|_{y=0}=\left.\Delta l_{z}\right|_{z=l_{z}}=\left.\Delta l_{z}\right|_{z=0}=0
\end{aligned}
$$

which means the displacement in $x$ axis is $0.001 l_{x}$ when $x=l_{x}$, i.e., $\varepsilon_{x}=0.001$ and the displacements in all other directions are zeros. The boundary conditions for shear strain cases were defined different. For example, in the case of shear strain $\gamma_{x y}\left(\varepsilon_{12}\right)$, the boundary conditions were

$$
\begin{aligned}
& \left.\Delta l_{x}\right|_{z=l_{x}}=0.0005 l_{z},\left.\Delta l_{z}\right|_{x=l z}=0.0005 l_{x} \\
& \left.\Delta l_{z}\right|_{x=0}=\left.\Delta l_{y}\right|_{y=l_{y}}=\left.\Delta l_{y}\right|_{y=0}=\left.\Delta l_{z}\right|_{z=l_{z}}=\left.\Delta l_{x}\right|_{z=0}=0
\end{aligned}
$$

Although the boundary conditions were defined only from mathematical viewpoint, this approach provides highly accurate results for predicting the macroscopic mechanical properties of materials and structures (Steven, 1997; Tan et al., 2000). The meshing size was sufficiently small so that the influence of the bending moment could be negligible. The whole homogenization procedure was coded in Fortran language with the finite element analysis of each numerical test conducted in ABAQUS.

Tridimensional orientation-dependent polar plots of Young's modulus surface are commonly used to represent the anisotropy of monocrystallines (Nye, 1985; Meyers and Chawla, 2009). Similar method may also be extended to characterize the non-continuum lattice structures after homogenization treatment of the compliance (or stiffness) matrix. In this work, the Young's modulus values at any direction are obtained through a succussive procedure used in classical books (Nye, 1985; Meyers and Chawla, 2009), including calculation of the direction cosine, transformation and rotation of stress tensor in different coordinate systems. The matrix of direction cosine $[\lambda](3 \times 3$ matrix) is predetermined when rotating unitary orthogonal coordinate

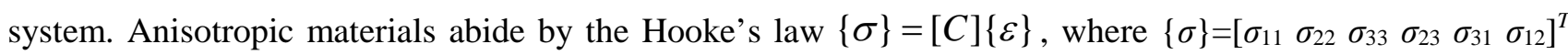
and $\{\varepsilon\}=\left[\begin{array}{lllllll}\varepsilon_{11} & \varepsilon_{22} & \varepsilon_{33} & \varepsilon_{23} & \varepsilon_{31} & \varepsilon_{12}\end{array}\right]^{T}$. After transformation in the orthogonal coordinate systems, the new stiffness matrix becomes $\left[C^{\prime}\right]=[T][C][T]^{T}$. The transformation matrix is dependent on the matrix of the direction cosine. Through this operation, the Young's modulus surface can be plotted in a 3D space at any direction defined by the direction cosine (different angles). The calculation was coded in Matlab to produce all the 3D Young's modulus surfaces in this work.

In this work, the stiffness matrix obtained through homogenization was firstly assessed to check the anisotropy. For isotropic structures, there are only two independent elastic constants, $C_{11}$ and $C_{12}$. In numerical analysis, the anisotropy of the structure can be measured by

$$
A=2 C_{44} /\left(C_{11}-C_{12}\right)
$$

If $A$ is close to unity, the structure could be treated as isotropic.

\section{Results and discussion}

\subsection{Anisotropy of lattice structures}

Unlike continuum metal materials, which are macroscopically isotropic due to the random distribution of crystalline grains, lattice structures are commonly elastically anisotropic with evident weak directions relating to the rod arrangement. Using numerical homogenization method proposed by Steven (Steven, 1997; Tan et al., 2000), we evaluated the effective stiffness matrix of a variety of reported representative unit cells (base units). Plotting the Young's modulus surface in an orthogonal coordinate system similar to the method for analysing the elastic anisotropy of monocrystallines (Nye, 1985; Meyers and Chawla, 2009), the strong and weak directions can be clearly demonstrated (Fig. 1). The elastic anisotropy of lattice structures is highly dependent on the spatial arrangement of rods. For example, the simple crossing-rod unit (Fig.1a) has much higher stiffness in diagonal directions than in axial directions. However, simple cubic unit (Fig.1b) is stiff in axial directions and weak in diagonal directions. Other structures such as face-centred cubic (FCC), diamond cubic, octet-truss unit and the combination of face-centred and body-centred (FCC-BCC) units show evident 
differences (Figs.1c to 1f). The spatial distribution of Young's modulus surfaces is only dependent on the rod arrangement and independent of the base material. Thus, for lattice structures, the dominant factor influencing the mechanical properties of structures is the spatial arrangement and dimension of the rods.

(a)
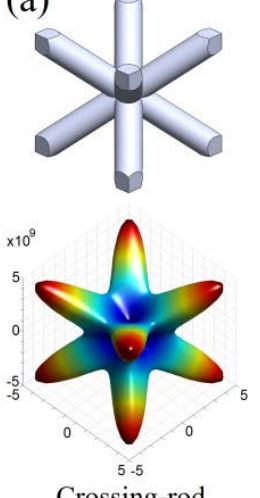

(d)

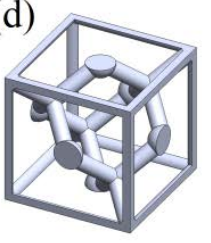

$\times 10^{8}$

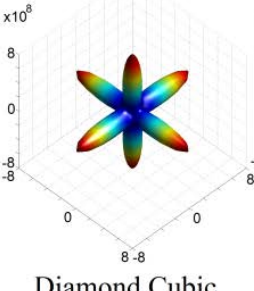

(b)

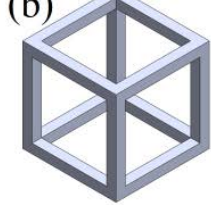

$\times 10^{\circ}$

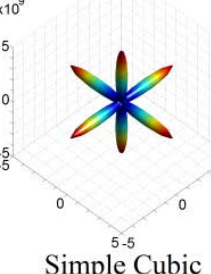

(e)
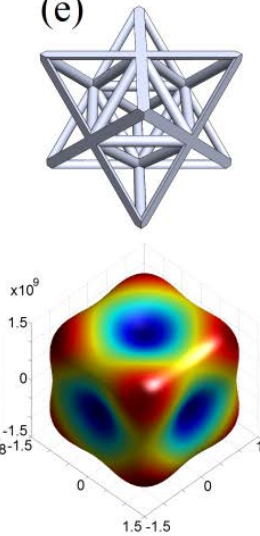

Octet-truss Unit

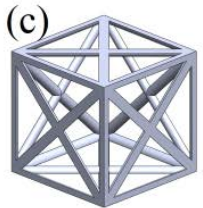

$\times 10^{\circ}$

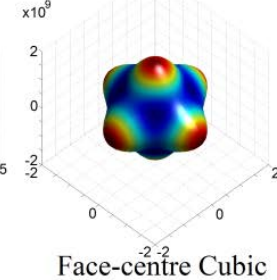

(f)
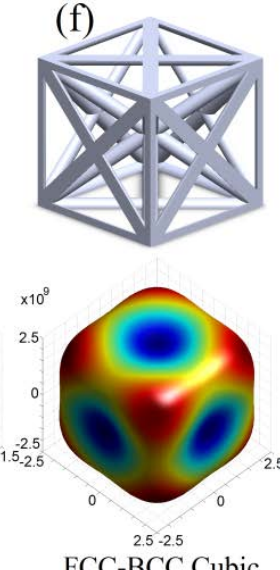

Fig. 1. Architectures of typical representative units and the corresponding 3D spatial representations of effective Young's modulus surfaces: (a) crossing-rod unit, (b) simple cubic unit, (c) face-centre cubic unit, (d) diamond cubic unit, (e) octet-truss unit, and (f) a combined unit of face-centred and body-centred units (FCC-BCC).

Anisotropy of structures is considered to be harmful in many applications, especially when anisotropic structures are used as structural components or energy absorption materials. For example, the Young's modulus surfaces of natural bones normally take ellipsoidal shapes, which can be plotted by applying the current 3D characterization method to the elasticity tensors of different bones from literature (Bernard et al., 2013). Hence ideal artificial replacements shall take similar Young’s modulus distributions as real bones with no obvious weak directions to avoid stress shielding, i.e., ellipsoidal or spherical (near-isotropic or isotropic). But, after an extensive evaluation using the 3D characterization method, we find that most reported artificial lattice structures for orthopaedic implantations are indeed anisotropic with significant discrepancy in different directions similar to those illustrated in Fig. 1. They normally have large ratio of the highest Young's modulus to the lowest, $E_{\max } / E_{\min }$. In applications such as energy absorption barrier of vehicles in impact events, the anisotropy of materials may also bring significant harm to occupants because of the decreased energy absorption capacity in weak directions. Hence, directly selecting simple representative units may not be a suitable approach for achieving optimal lattice designs. It is therefore necessary to develop proper methodologies to design lattice structures with controlled anisotropy that could fulfil the specific needs in various applications.

\subsection{Methodology to design elastically isotropic lattice structures}

In this work, three types of representative units were proposed and examined after analysing their elastic mechanical properties, namely FCC-BCC unit (Fig. 2a), diamond cubic (Fig. 2b) and complex diamond cubic 
(CDC, Fig. 2c), the materials parameters were defined as: Young's modulus $E_{s}=153 \mathrm{GPa}$ for $\mathrm{ALD} \mathrm{Al}_{2} \mathrm{O}_{3}$ (Zheng et al., 2014) and Poisson's ratio $v=0.3$.
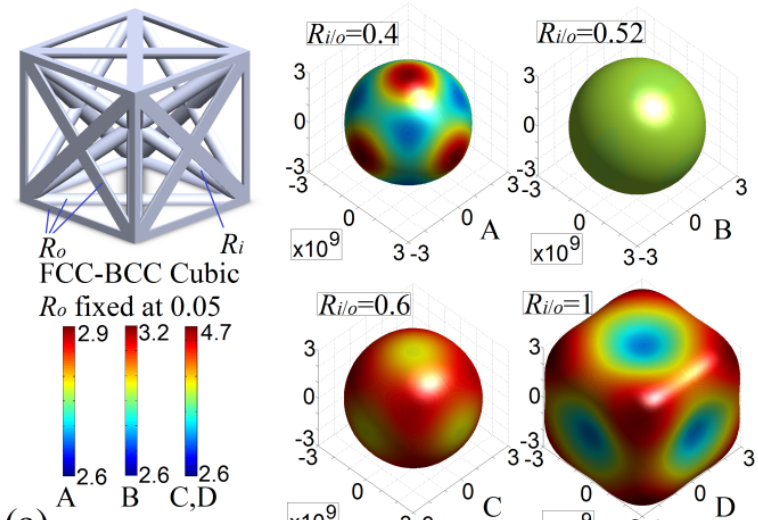

(a)
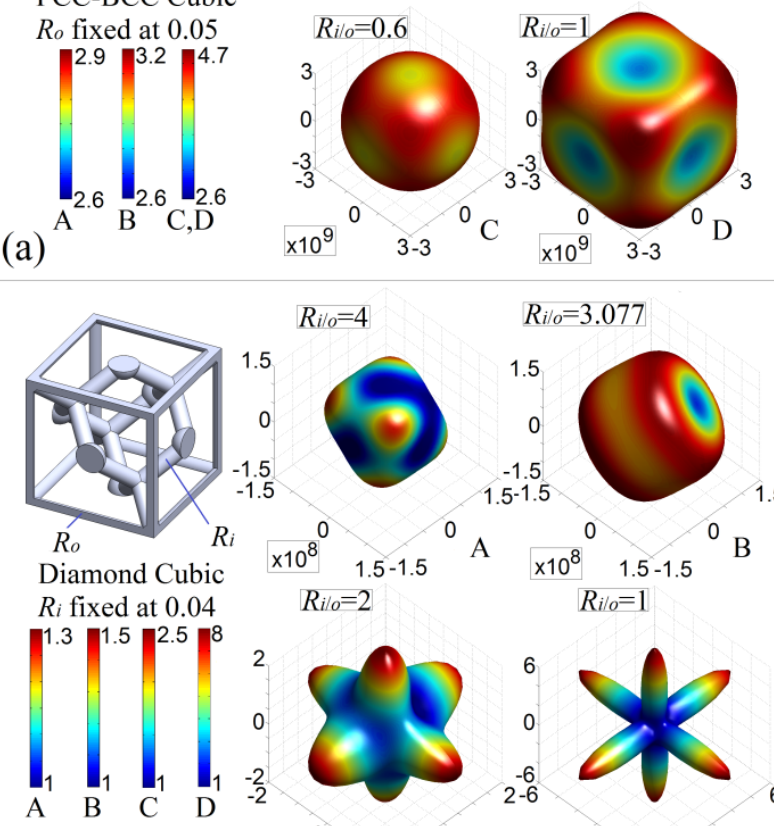

$R_{i / 0}=4 \quad \quad R_{i l}=3.077$

(b)
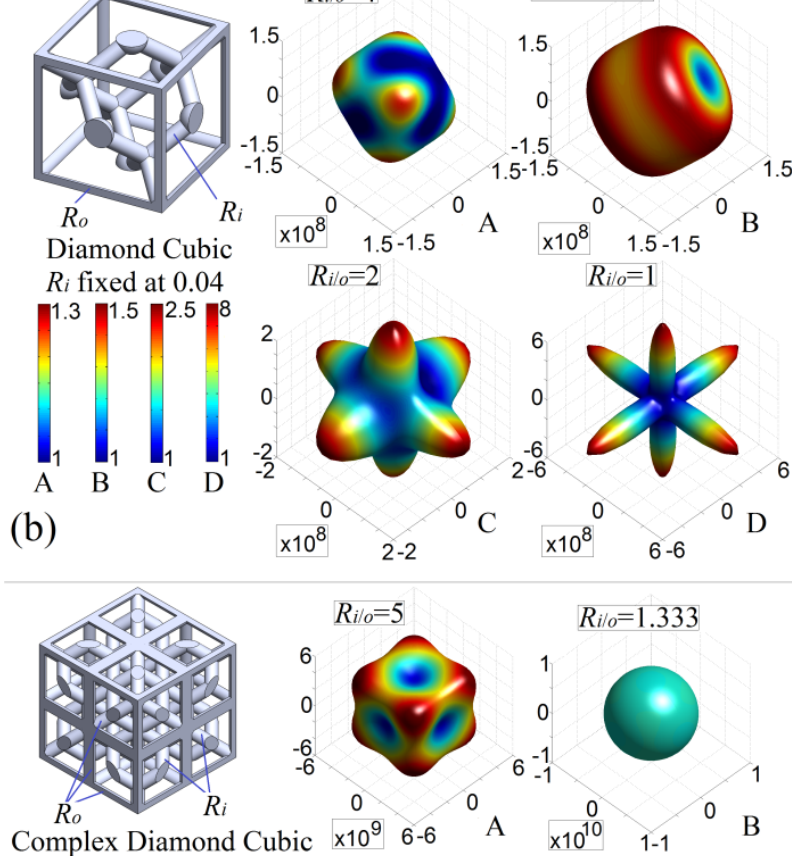

(CDC) $R_{i}$ fixed at 0.05

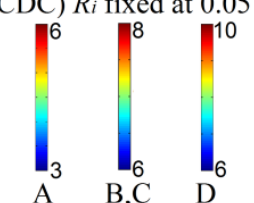

(c)

$R_{i / o}=1.111 \quad \quad R_{i / 0}=1$

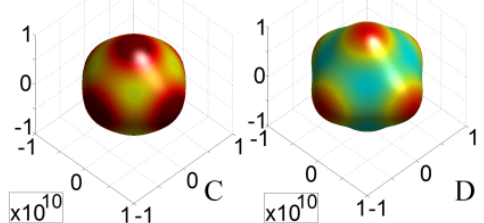

Fig. 2. Obtaining 2D and $3 \mathrm{D}$ isotropic structures via different methods: (a) combination of base units with complementary stiffness in inverse directions, a FCC-BCC structure, (b) diamond cubic and (c) mirror operation on a diamond cubic unit to obtain a complex diamond cubic (CDC) which becomes isotropic after changing the ratio of $R_{i} / R_{0}$.

From the previous assessment results, a seemingly obvious choice to achieve controlled anisotropy is to combine basic structural units with complementary Young's modulus distributions in spatial directions. For example, crossing-rod (Fig. 1a) and FCC (Fig. 1c) units could be easily jointed and could also compensate the stiffness in reverse directions each other to form a FCC-BCC structure. It shall be noted that those rods in diagonal directions contribute partially to the stiffness in both diagonal and axial directions, but with different shares. Hence, it is possible to re-distribute the Young's modulus values in different directions by simply adjusting the ratios of rod diameters in different regimes of the unit. Therefore, the problem turns to be a case of parametric study. In the newly built FCC-BCC cubic, taking the rod diameter of the crossing-rod as $R_{i}$ and 
FCC unit as $R_{o}$. Adjusting $R_{i / o}=R_{i} / R_{o}$ from 0.4 to 1 with $R_{o}$ (radius of inside diagonal rods) fixed at 0.05 in a unit cell, the evolution of 3D Young's modulus surface is demonstrated in Fig. 2a. With the increase of $R_{i / o}$, the absolute values of Young's modulus increase. In the meantime, the ratio of the maximum $\left(E_{\max }\right)$ to the minimum $\left(E_{\min }\right)$ effective Young's modulus, $E_{\max / \min }=E_{\max } / E_{\min }$, drops from 1.082 at $R_{i / o}=0.4$ to 1.001 at $R_{i / o}=0.52$, then increase to 1.496 at $R_{i / o}=1$. Through this strategy, we successfully obtained an elastically isotropic design of lattice structures when $R_{i / o}=0.52$, the Young's modulus surface becomes almost spherical. It should be noted that when adjusting the $R_{i / o}$ values, the symmetry of the structure shall not be altered. Otherwise isotropic properties could not be obtained. In this work, only cubic units were analysed. When extending one of its edges to form a tetragonal unit, it is likely to obtain an ellipsoidal shape in terms of the Young's modulus surface through the same methodology. It should be pointed out that this method cannot consequently lead to a design with isotropic elasticity, depending on how the two base units compensate each other.

Another idea to design elastically isotropic structures may naturally direct to adjusting the ratios of rod diameters in different directions since rods in a unit are arranged in diagonal or axial directions as shown in Figs. 1c to 1f. However, we find it difficult to obtain elastically isotropic structures by this type of simple operation. The octet-truss unit, which is a well-known stretch dominant structure, could be decomposed into two components, the inside octahedron unit enclosed by a cubic frame. However, changing the dimension of rods will not bring any change of the spatial distribution of Young's modulus surface. For FCC unit, the number of rods in one diagonal direction is always less than that in axial directions. Therefore, the principal shape of Young's modulus surface will be kept unchanged.

An interesting case comes from the diamond cubic unit (Fig. 1d). This unit is claimed to be isotropic by some researchers without the rods in the three axial directions (Ahmadi et al., 2014). Due to the asymmetry feature of this structure, small balls are included at the centre of the six cubic faces, which could just cover the joint region of those diagonal rods and have limited influence on the principal shape of the Young's modulus. When decreasing the $R_{i / o}$ values from 4 to 3.077 with $R_{i}$ (radius of diagonal rods) fixed at 0.04 in a unit cell, the Young's modulus surface reaches a near-cylindrical shape with the central axis in direction [111] (Fig. 2b). In the (111) plane, the Young's modulus exhibits an almost identical value about the rotational axis [111], i.e., a type of two-dimensional isotropy in the plane perpendicular to [111] axis. With the decrease of $R_{i / o}$ from 3.007 to 1 , this type of isotropy is lost. This interesting phenomenon is believed to be from the geometrical asymmetry of the structure, which is only mirror symmetry about (110) and (011) planes.

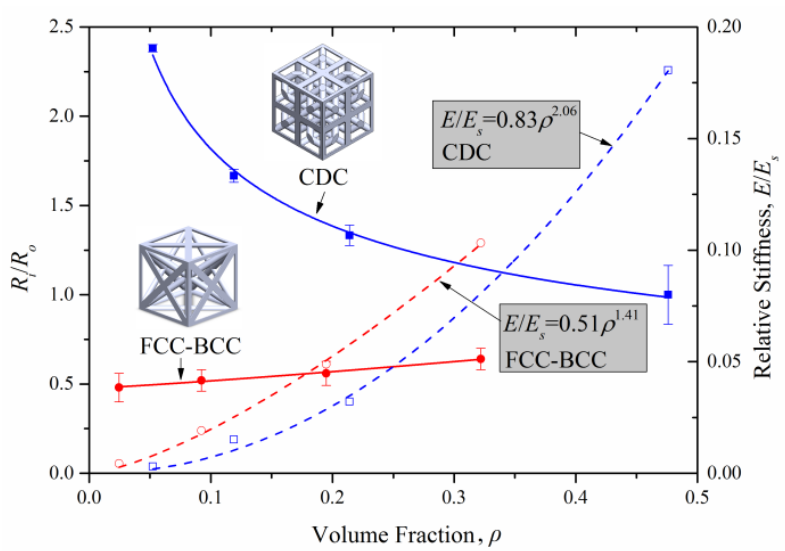

Fig. 3. The change in $R_{i} / R_{o}$ ratio with the increasing volume fraction of two types of representative units, CDC and FCC-BCC, by fixing the diameter of one type of rod and adjusting another type (left vertical axis, solid lines). The relative stiffness (E/Es) of the two structures in relation to the volume fraction (right vertical axis, dashed lines).

Inspired by the analysis on the diamond cubic unit, a new design strategy can be proposed to bring controlled anisotropy of structures. Noticing the two-dimensional isotropic feature of diamond unit, it is possible to design such structures that are arrayed through symmetry operation to form a new structure, which 
is composed of multiple base units with limited geometrical symmetry in certain directions. For example, through a series of mirror operations on the diamond unit, a fully symmetrical structural unit containing eight basic units could be built (Fig. 2c). By fixing one rod diameter and changing the ratio between rod diameter of the diagonal lattice $\left(R_{i}\right)$ and the rectangular frame $\left(R_{o}\right), R_{i} / R_{o}$ (also defined as $R_{i / o}$ for convenience), we successfully obtained another structure with elastic isotropy when $R_{i / o}$ decreasing from 5 to 1.333 , with the corresponding $E_{\max / \min }=1.009$.

However, it has to be pointed out that when the rod diameter is fixed at another value, the anisotropy of the entire structure changes. New $R_{i} / R_{o}$ ratio to achieve isotropy has to be re-evaluated. Therefore, it will, indeed, generate a group of elastically isotropic structures with varying volume fractions (Fig. 3). The lattice type will affect the variation of $R_{i} / R_{o}$ ratio, as well as the adaptability, i.e., the range for the structure to remain almost isotropic (variation of anisotropy coefficient within $\pm 5 \%$ ). For CDC structure, $R_{i} / R_{o}$ decreases with the increase of volume fraction. While for FCC-BCC structure, it decreases. In the meantime, the adaptability of the two structures is different. The range of $R_{i} / R_{o}$ ratio for CDC structures is much more flexible at higher volume fraction while significantly narrowed at lower volume fractions. On the contrary, FCC-BCC structure remains an almost constant and moderate $R_{i} / R_{o}$ range, which makes it much easier to achieve isotropic properties over the studied volume fraction values in manufacturing. It can also be seen in Fig. 3 that the relative stiffness for FCC-BCC structures (the ratio of structure stiffness to stiffness of base materials, $E / E_{s}$ ) when $E_{s}=153 \mathrm{GPa}$ for $\mathrm{ALD} \mathrm{Al}_{2} \mathrm{O}_{3}$ (Zheng et al., 2014) are higher than that for CDC structures. The dependence of relative stiffness on the volume fraction, $\rho$, is in power relations with exponent 1.41 for FCCBCC and 2.06 for CDC lattice structures.

These two strategies provide significant privilege to find elastically isotropic periodical structures through a simple preliminary assessment followed by a parametric study. The whole procedure could easily be programmed and calculated by computers. Some researchers have tried to use topological optimization method such as bi-directional evolutionary structural optimization (BESO) developed by Xie and colleagues (Xie and Steven, 1997; Huang and Xie, 2010) to obtain structures with isotropic stiffness matrix (Yang et al., 2013). New lattice structures could be designed by simplifying the obtained topologies from these techniques according to specific loading cases.

\section{Conclusions}

Conventional design of lattice structures for bone implants and other applications with specified mechanical properties normally pays little attention to the anisotropic properties, which is in fact indispensable. The present work proposed new strategies to address this issue by integrating the homogenization method and 3D characterization of Young's modulus surface. Two approaches were proposed for designing lattice structures with controlled anisotropy. One is to assemble two different base units with complementary stiffness along various spatial directions to form a new representative unit. Then, by adjusting the ratio of rod diameters between the two base units, a controlled anisotropy could be achieved (typically isotropic). The other is to employ one single base unit with partially symmetrical features and array it through symmetry operations to construct a new unit which is composed of multiple original base units. Then, by adjusting the ratio of rod dimensions between different regimes of the base unit, controlled anisotropic features could also be achieved. Two groups of lattice structures with elastically isotropic properties were proposed by using these two strategies. The anisotropy of lattice structures is highly related to the spatial arrangement and dimensions of rods. When changing rod dimensions in one regime, the anisotropy of the entire structure will be altered. A new ratio of dimensions between rods in different regimes has to be evaluated in order to reach the desired anisotropy.

\section{Acknowledgement}


This work was supported by the Australian Research Council (DP140100213), the National Natural Science Foundation of China (51228801) and the Open Research Fund Program of the State Key Laboratory of Advanced Design and Manufacturing for Vehicle Body (41215001).

\section{References}

Ahmadi, S.M., Campoli, G., Amin Yavari, S., Sajadi, B., Wauthle, R., Schrooten, J., Weinans, H., Zadpoora, A.A., 2014. Mechanical behavior of regular open-cell porous biomaterials made of diamond lattice unit cells. J. Mech. Behav. Biomed. Mater. 34, 106-115.

Bäeuerlein, E., Behrens, P., Epple, M., 2007. Handbook of biomineralization: biological aspects and structure formation, Wiley-VCH.

Bernard, S., Grimal, Q., Laugier, P., 2013. Accurate measurement of cortical bone elasticity tensor with resonant ultrasound spectroscopy. J. Mech. Behav. Biomed. Mater. 18, 12-19.

Challis, V.J., Roberts, A.P., Grotowski, J.F., Zhang, L.C., Sercombe T.B., 2010. Prototypes for bone implant scaffolds designed via topology optimization and manufactured by solid freeform fabrication. Adv. Eng. Mater. 12, 1106-1110.

Chantarapanich, N., Puttawibul, P., Sucharitpwatskul, S., Jeamwatthanachai, P., Inglam, S., Sitthiseripratip, K., 2012. Scaffold library for tissue engineering: a geometric evaluation. Comput. Math. Methods Med. 2012, 407805

Evans, A.G., He, M.Y., Deshpande, V.S., Hutchinson, J.W., Jacobsen, A.J., Carter, W.B., 2010. Concepts for enhanced energy absorption using hollow micro-lattices. Int. J. Impact. Eng. 37, 947-959.

Giannitelli, S.M., Accoto, D., Trombetta, M., Rainer, A., 2014. Current trends in the design of scaffolds for computer-aided tissue engineering. Acta Biomater. 10, 580-594.

Gibson, I., Rosen, D.W., Stucker, B., 2010. Additive manufacturing technologies, Springer.

Gibson, L.J., Ashby, M.F., 1991. Cellular solids: structure and properties, Cambridge University Press, Cambridge.

Guo, N., Leu, M.C., 2013. Additive manufacturing: Technology, applications and research needs. Front. Mech. Eng. 8, 215-243.

Gurtner, G., Durand, M., 2014. Stiffest elastic networks. Proc. R. Soc. A. 470, 20130611.

Heinl, P., Müller, L., Körner, C., Singer, R.F., Müller, F.A., 2008. Cellular Ti-6Al-4V structures with interconnected macro porosity for bone implants fabricated by selective electron beam melting. Acta Biomater. 4, 1536-1544.

Huang, X., Xie, Y.M., 2010. Evolutionary topology optimization of continuum structures: methods and applications, John Wiley and Sons.

Hutmacher, D.W., 2000. Scaffolds in tissue engineering bone and cartilage. Biomaterials. 21, 2529-2543.

Karageorgiou, V., Kaplan, D., 2005. Porosity of 3D biomaterial scaffolds and osteogenesis. Biomaterials. 26, 5474-5491.

Liu, C., Li, F., Lai-Peng, M., Cheng, H.M., 2010. Advanced materials for energy storage. Adv. Mater. 22, E28-E62.

Meyers, M.A., Chawla, K.K., 2009. Mechanical behavior of materials, Cambridge University Press, Cambridge.

Meza, L.R., Das, S., Greer, J.R., 2014. Strong, lightweight, and recoverable three-dimensional ceramic nanolattices. Science. 345, 1322-1326.

Murphy, S.V., Atala A., 2014. 3D bioprinting of tissues and organs. Nat. Biotechnol. 32, 773-785.

Murr, L.E., Gaytan, S.M., Medina, F., Lopez, H., Martinez, E., et al., 2010. Next-generation biomedical implants using additive manufacturing of complex cellular and functional mesh arrays. Phil. Trans. R. Soc. A. 368, 1999-2032.

Nye, J., 1985. Physical properties of crystals: their representation by tensors and matrices. Oxford University Press, Oxford.

Parthasarathy, J., Starly, B., Raman, S., Christensen, A., 2010. Mechanical evaluation of porous titanium (Ti6Al4V) structures with electron beam melting (EBM). J. Mech. Behav. Biomed. Mater. 3, 249-259.

Round, F.E., Crawford, R.M., Mann, D.G., 1990. The diatoms: biology \& morphology of the genera, Cambridge University Press, Cambridge.

Sallica-Leva, E., Jardini, A.L., Fogagnolo, J.B., 2013. Microstructure and mechanical behavior of porous Ti6Al-4V parts obtained by selective laser melting. J. Mech. Behav. Biomed. Mater. 26, 98-108. 
Schaedler, T.A., Jacobsen, A.J., Torrents, A., Sorensen, A.E., Lian, J., Greer, J.R. Valdevit, L. Carter, W.B., 2011. Ultralight metallic microlattices. Science. 334, 962-965.

Steven, G.P., 1997. Homogenization of multicomponent composite orthotropic materials using FEA. Numer. Methods Eng. 13, 517-531.

Tan, P., Tong, L., Steven, G.P., 2000. Behavior of 3D orthogonal woven CFRP composites. Part II. FEA and analytical modeling approaches. Compos. Part. A. 31, 273-281.

Wadley, H.N.G., 2006. Multifunctional periodic cellular metals. Philos. Trans. R. Soc. A. 364, 31-68.

Wieding, J., Wolf, A., Bader, R., 2014. Numerical optimization of open-porous bone scaffold structures to match the elastic properties of human cortical bone. J. Mech. Behav. Biomed. Mater. 37, 56-68.

Xie, Y.M., Steven, G.P., 1997. Evolutionary structural optimization, Springer.

Xie, Y.M., Yang, X., Shen, J., Yan, X., Ghaedizadeh, A., Rong, J., Huang, X., Zhou, S., 2014. Designing orthotropic materials for negative or zero compressibility. Int. J. Solids Struct. 51, 4038-4051.

Xie, Y.M., Zuo, Z.H., Huang, X., Yang, X., 2013. Predicting the effective stiffness of cellular and composite materials with self-similar hierarchical microstructures. J. Mech. Mater. Struct. 8, 341-357.

Yang, X.Y., Huang, X., Rong, J.H., Xie, Y.M., 2013. Design of 3D orthotropic materials with prescribed ratios for effective Young's moduli. Comput. Mater. Sci. 67, 229-237.

Zheng, X., Lee, H., Weisgraber, T.H., Shusteff, M., DeOtte, J., Duoss, E.B., Kuntz, J.D., Biener, M.M., Ge, Q., Jackson, J.A., Kucheyev, S.O., Fang, N.X., Spadaccini, C.M., 2014. Ultralight, ultrastiff mechanical metamaterials. Science. 344, 1373-1377. 